\title{
Total Hip Replacement and Distal Femur Shaft Non-union in a Case of Below-knee Amputation - A Case Report
}

\author{
Eknath Pawar ${ }^{1}$, Sandeep Gavhale ${ }^{1}$, Mrinal Kambli $^{1}$, Amit Yadav ${ }^{1}$, Sagar Bansal ${ }^{1}$, Shaswat Mishra ${ }^{1}$
}

\section{Learning Point of the Article:}

Total hip replacement with distal femur plating and allogenic bone grafting can be used as a primary and definitive modality of treatment in cases of neck of femur fractures with distal femur shaft non-union and ipsilateral below-knee amputation.

\section{Abstract}

Introduction: Primary total hip replacement (THR) surgery for the neck of femur (NOF) fracture in a case of below-knee amputation with distal femur shaft non-union is rare.

Case Report: This case describes the traumatic right NOF fracture in a 49-year-old male patient with distal femur shaft non-union. The patient has a history of ipsilateral below the knee amputation along with right distal femur shaft fracture managed with retrograde femur nailing 10 months back following a road traffic accident. The NOF fracture was managed by primary THR as the definitive procedure after distal femur nail removal and distal femur plating with allograft for non-union.

Conclusion: Primary THR with distal femur plating and allogenic bone grafting in such cases provides satisfactory outcomes. It allows for early ambulation and rehabilitation of the patient.

Keywords: Primary total hip replacement, Below-knee amputation, Distal femur non-union.

\section{Introduction}

Neck of femur (NOF) fractures has been a major cause of morbidity and mortality throughout the world, but the development of hip arthroplasty has helped reduce this morbidity, improve the survival rate, and start early ambulation and rehabilitation for such patients. Although the procedure is a fairly common practice, its use in ipsilateral below-knee amputation with non-united distal femur shaft fracture patients has been seldom seen. Management of such a case is challenging as it poses several technical difficulties including patient setup, stump manipulation, approach, and surgical techniques. It requires extensive pre-operative planning to avoid any intraoperative or post-operative complications.

Here, we have presented a case of primary total hip replacement (THR) in a below-knee amputation and distal femur plating with allogenic bone grafting in a 49-year-old male.

\section{Case Report}

We present a 49-year-old man, with no comorbidities. The patient had a history of a road traffic accident 10 months ago when the patient had to undergo below-knee amputation at a peripheral center for distal one-third tibia fibula shaft fracture Gustilo Anderson Grade IIIc [1]. At the same time, the patient also had a distal femur shaft fracture (Fig. 1) which was operated with retrograde femur nailing (Fig. 2). The patient was mobilized with crutches. Seven months following the belowknee amputation, the patient had a history of fall at home. The patient presented to our hospital with pain in the right hip and restricted range of motion 3 months post-trauma. A standard anteroposterior radiograph of pelvis with both hips showed right NOF fracture and non-union of the right distal femur shaft fracture with a femoral nail in situ (Fig. 3). Pre-operative planning was done to avoid intraoperative or post-operative Author's Photo Gallery

\begin{tabular}{|c|}
\hline Access this article online \\
\hline Website: \\
www.jocr.co.in \\
\hline DOI: \\
10.13107/jocr.2021.v11.02.2046 \\
\hline
\end{tabular}
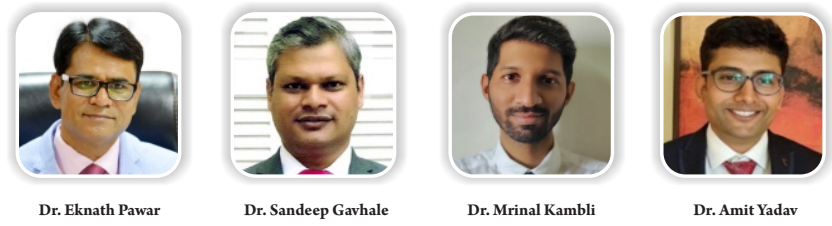

Dr. Sandeep Gavhale $\quad$ Dr. Mrinal Kambli

Dr. Amit Yadav

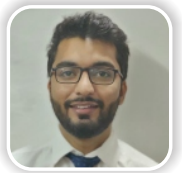

Dr. Sagar Bansal

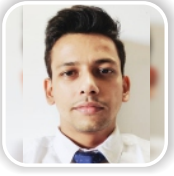

Dr. Shaswat Mishra
${ }^{1}$ Department of Orthopaedics, Grant Medical College and Sir J.J. Group of Hospitals, Byculla, Mumbai, Maharashtra, India.

Address of Correspondence:

Dr. Shaswat Mishra,

Department of Orthopaedics, Grant Medical College and Sir J.J. Group of Hospitals, Byculla, Mumbai, Maharashtra, India.

E-mail: shaswatmishra1994@gmail.com

Journal of Orthopaedic Case Reports | pISSN 2250-0685 | eISSN 2321-3817 | Available on www.jocr.co.in | doi:10.13107/jocr.2021.v11.i02.2046 This is an Open Access article distributed under the terms of the Creative Commons Attribution Non-Commercial License (http://creativecommons.org/licenses/by-nc/3.0) which permits unrestricted non-commercial use, distribution, and reproduction in any medium, provided the original work is properly cited. 


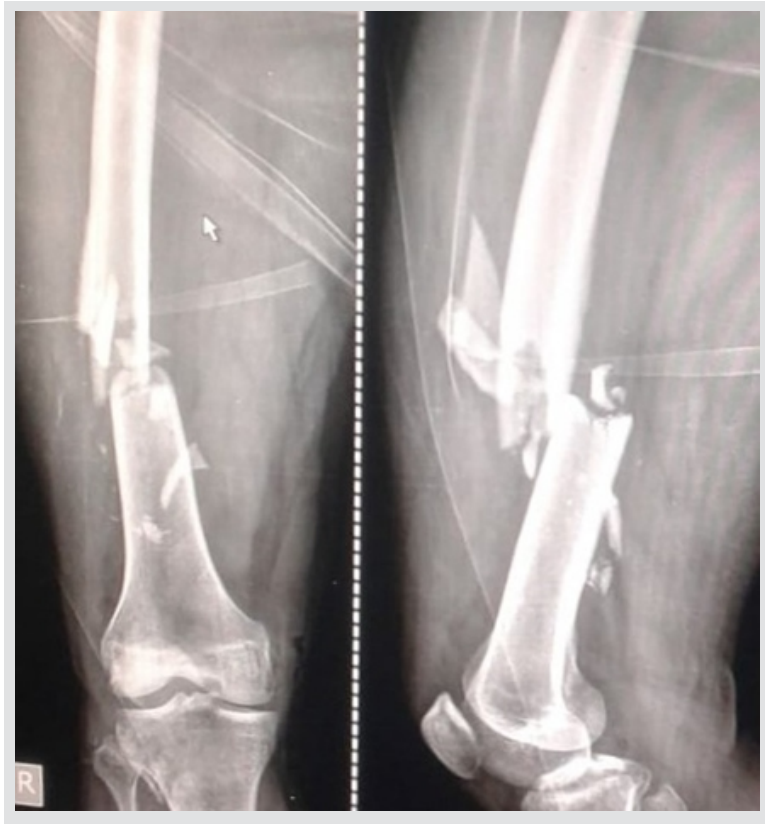

Figure 1: Pre-operative anteroposterior and lateral radiographs of the distal femur shaft fracture.

complications. Distal femur nail was removed and compression plating through lateral approach with allogenic bone grafting (Fig. 4) and uncemented right THR surgery (Fig. 5) done through Moore approach as the definitive procedure for the patient.

\section{Surgical technique}

The patient first placed supine on the operating table with the knee of the amputated limb in $30^{\circ}$ flexion. The distal femoral nail was removed, the patient then shifted to a lateral decubitus position and secured with lateral supports to prevent rotation from a true lateral position. Using Moore's approach, uncemented primary THR was done [2]. The patient was kept in the same position and distal femur plating was done using a lateral approach to distal femur fractures. Fixation was done with low contact dynamic compression plate. Allogenic bone

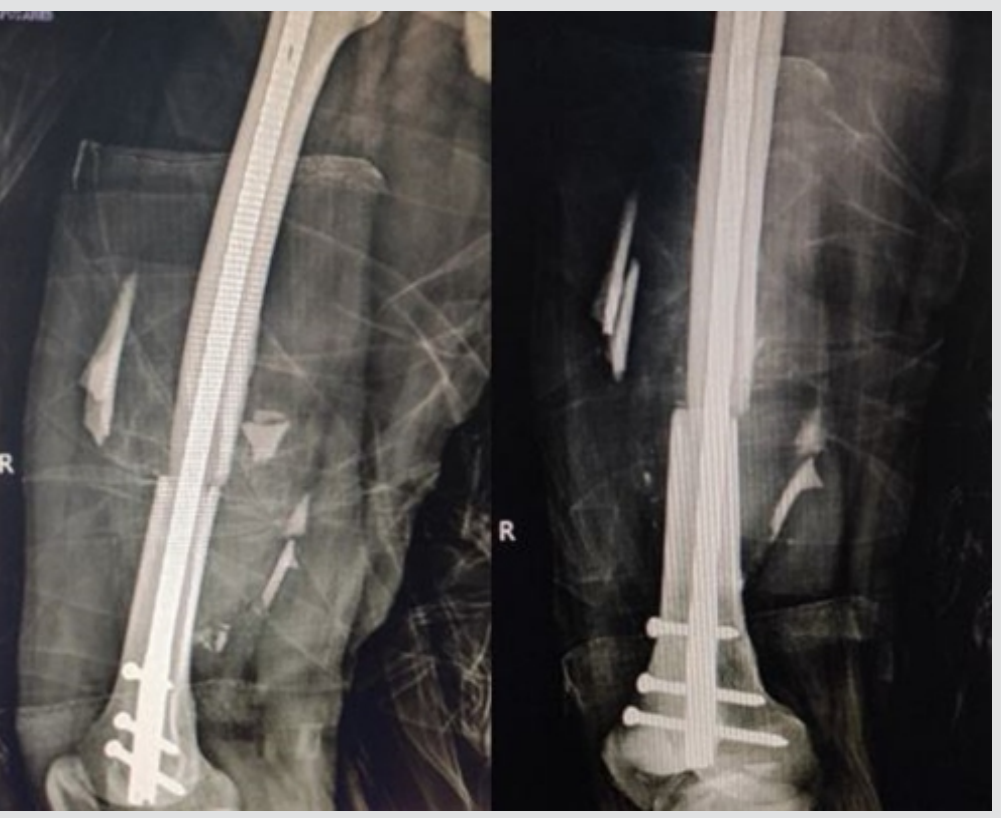

Figure 2: Post-operative anteroposterior and lateral radiograph after retrograde femur nailing.

grafting was done to promote union at the fracture site as it is a favorable and convenient alternative to autograft and also lacks donor site morbidity [3]. Bedside physiotherapy including hip and knee range of motion exercises, static hamstring, and quadriceps strengthening exercises started postoperatively on day 2 . The patient advised nil weight-bearing for 3 weeks. After 3 weeks, the patient started assisted walk with a walker with partial weight-bearing using a prosthesis. After 8 weeks postoperative, assisted walk with a walker with full weight-bearing started. Now 1-year post-surgery, the patient has no pain and can walk unassisted using prosthesis (Fig. 6). Follow-up radiographs (Fig. 7) at 6 months revealed a radiological union seen at the distal femur shaft non-union site. Range of motion is free and full at both hip and knee joints (Fig. 8)

\section{Discussion}

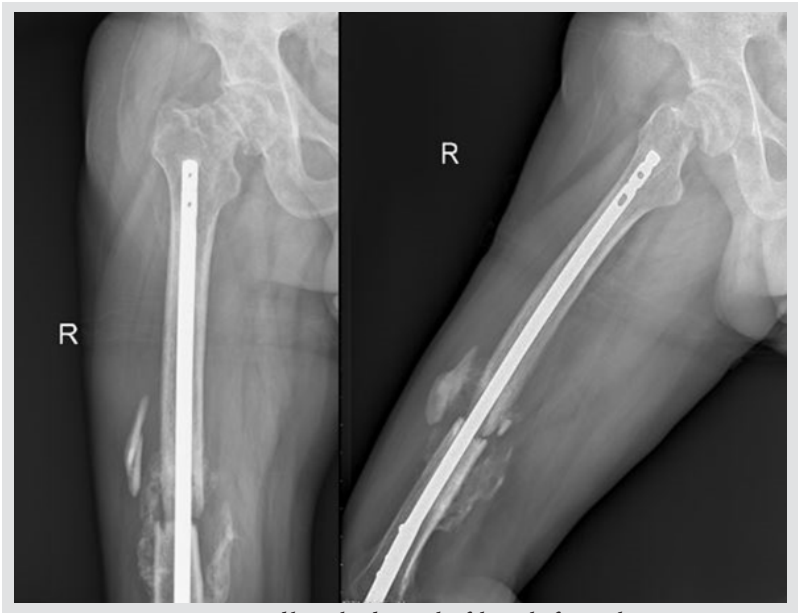

Figure 3: Anteroposterior and lateral radiograph of the right femur showing non-union at the fracture site with femoral nail in situ.

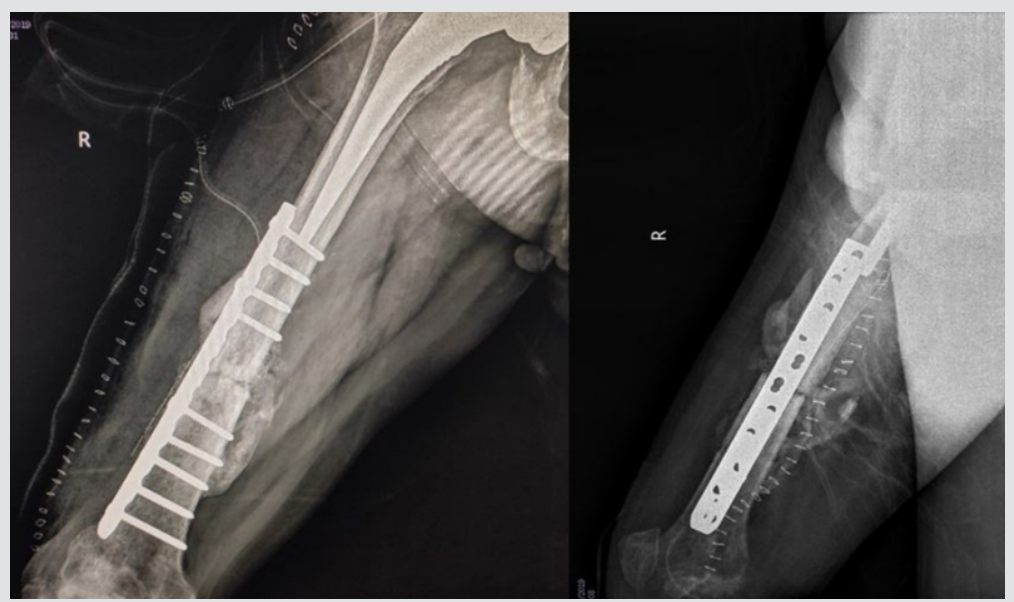

Figure 4: Post-operative anteroposterior and lateral radiograph of the right femur showing distal femur plating with bone grafting. 


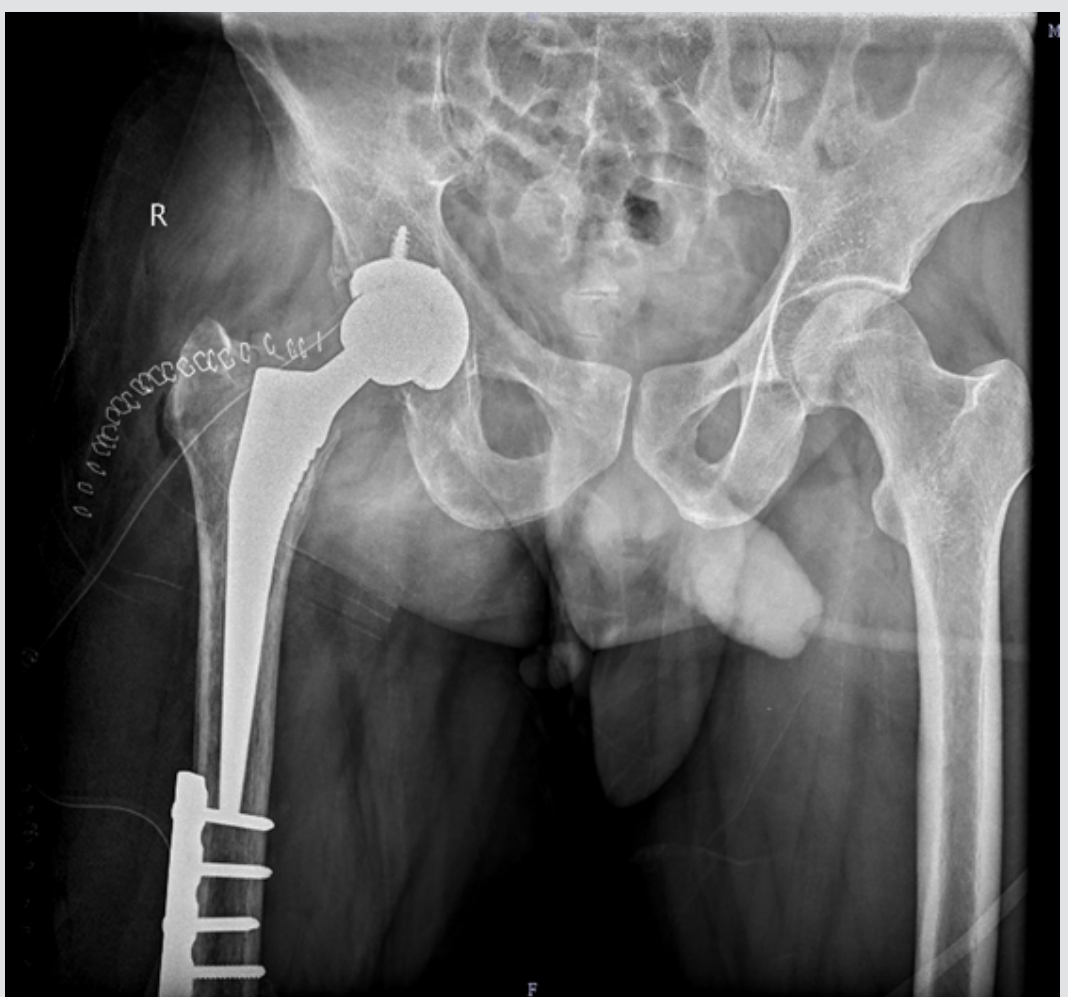

Figure 5: Post-operative anteroposterior radiograph of the pelvis after total hip replacement.

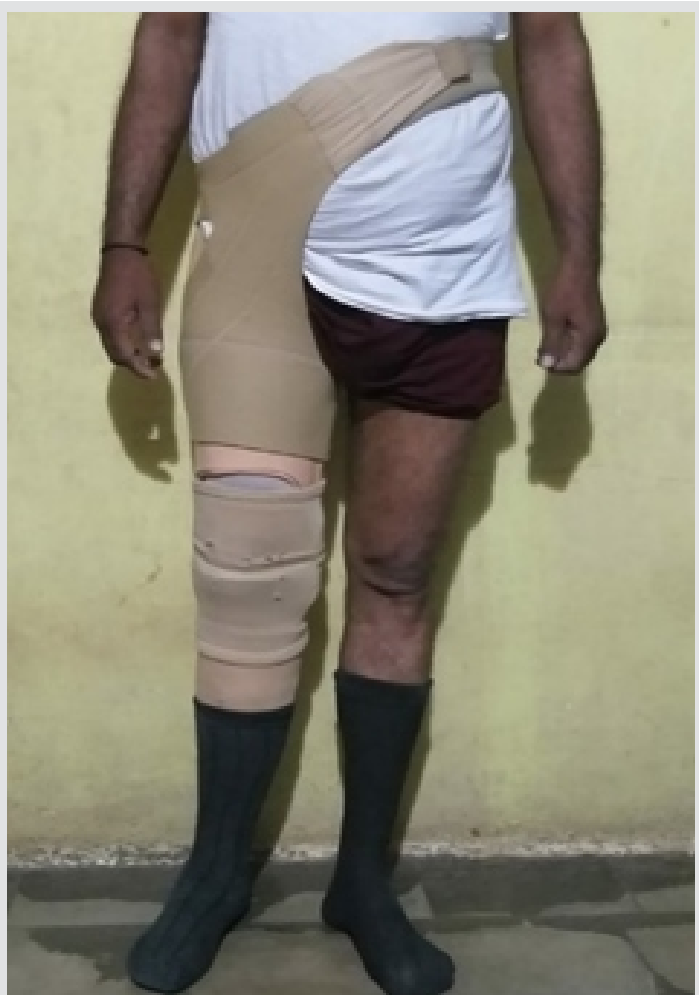

Figure 6: The clinical picture with a prosthesis at 6 months follow-up.
The NOF fractures have been a major cause of morbidity and mortality worldwide, especially for elderly population [4]. The advent of THR and its constant evolution has improved the prognosis in these fractures drastically. The NOF fracture with distal femur non-union in a case of below-knee amputation is rarely encountered and insufficient literature is available regarding the subject $[5,6]$. On long-term follow-up of belowknee amputation cases, it has been observed that there is an increase in knee osteoarthritis both patellofemoral and tibiofemoral, increase in ipsilateral hip arthritis, increase in osteopenia/osteoporosis of the amputated limb, and both amputated and normal side show amyotrophy [7]. It may be due to the surgery and loss of muscle insertions or myodesis of the muscles at a non-physiological site [8]. These may cause an increased incidence of residual fractures in the amputated limb. Reduction and fixation of such fractures can be attempted, especially in relatively young patients, although there is considerable risk of non-union with this modality. It can be explained with the lateral shift of the center of gravity for these patients which has increased the shearing forces acting across the fracture site [9]. A few publications are available stating the use of primary THR for the NOF fractures in ipsilateral aboveknee amputation. However, none of them had non-united distal one-third femur shaft fracture as in this case.

Salai et al. reported a series of five patients who underwent traumatic below-knee amputation and required THR for ipsilateral subcapital NOF fracture. Primary THR was done for two out of these five patients and THR was done as a secondary procedure for the other three patients after failure of closed reduction and internal fixation [5]. Masmoudi et al. suggested the use of primary THR for an acute NOF fracture in belowknee amputation, before the onset of avascular necrosis of femoral head and hip osteoarthritis. THR also proved better rehabilitation with early full weight-bearing and return to

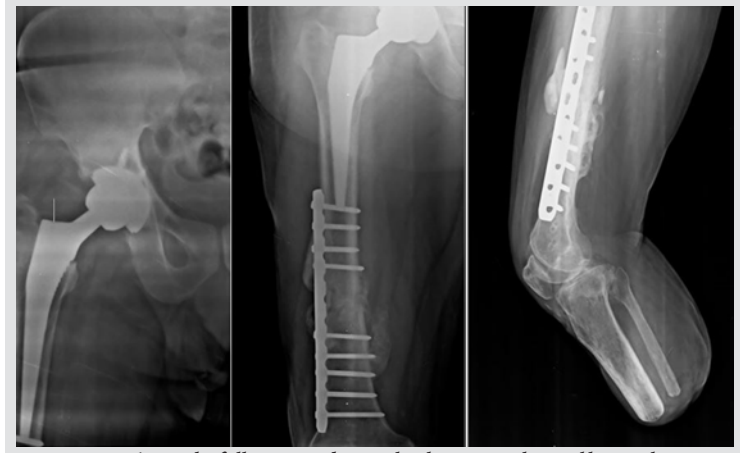

Figure 7: At 6 months follow-up radiographs showing right total hip replacement and union at distal femur.

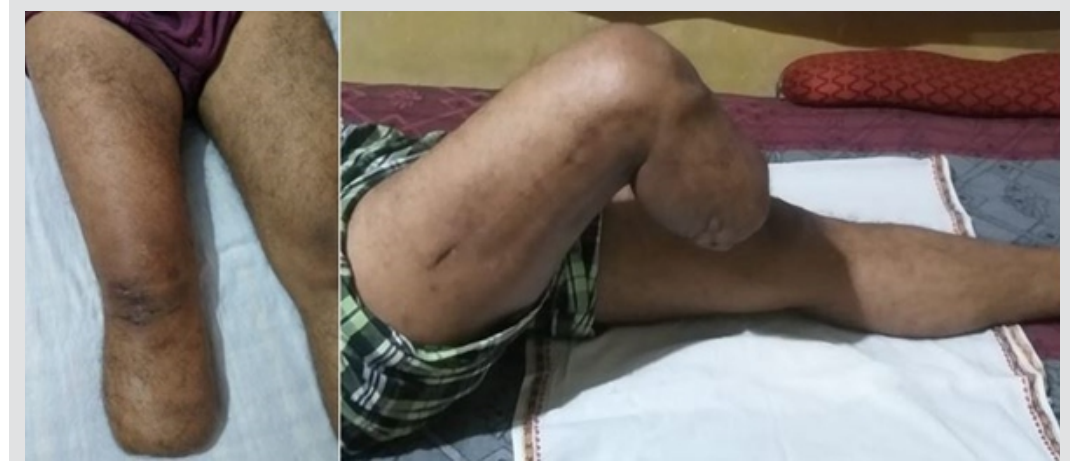

Figure 8: Clinical pictures of hip and knee range of motion 6 months post-operative. 
ambulation, unlike internal fixation [10]. Pekmezci et al. described the surgical technique for THR in a patient with below-knee amputation. The patient was taken in a lateral decubitus position and secured using a pegboard. Traction was applied to the operative limb by passing a $5 \mathrm{~mm}$ Steinman pin through the distal femur shaft because the femoral condyles were osteoporotic and covered by scar tissue. The standard horseshoe traction apparatus was attached to this pin [9]. Boussakri et al. described hip arthroplasty in a patient with a transfemoral amputation using a lateral approach of Hardinge which has an advantage of better exposure for alignment of the implant. This allowed it to be less traumatic and provided rotation and traction of the stump, thereby also reducing the surgery time compared to Moore and Kocher-Langenbeck approach $[6,13]$.

Bipolar hemiarthroplasty has been used as an alternative to THR in neck femur fractures frequently, but primary THR provides a better outcome with lesser pain and morbidity as compared to bipolar hemiarthroplasty [14]. It also avoids the need of a second procedure, that is, THR in the future. This is a rare case report of THR with distal femur compressive plating and allogenic bone grafting in a patient with the NOF fracture with ipsilateral below-knee amputation with distal femur shaft non-union. In this case, we chose a primary THR because of delayed presentation of a neck fracture, the higher life expectancy of the patient (no comorbidities), the higher level of activity of the patient and the need for a single definitive procedure for the patient as the patient had already been operated multiple times before.

In this case, we did not need to use the specific materials and methods described above as the patient had a NOF fracture so there was no need for hip dislocation. The patient had a belowknee amputation so the knee (popliteal fossa) was used as a fulcrum. The required traction could be given using the knee (popliteal fossa) as a fulcrum; so the use of skeletal traction using Steinman pins or bone holder was not needed thus avoiding damage to the already osteopenic/osteoporotic bone.

Physical therapy needs to be modified minimally for the patients with total joint replacements in extremities with belowknee amputations compared to other patients [11]. The Postoperative rehabilitation of an amputee with an ipsilateral hip replacement requires taking into account the likely development of contractures at the hip and knee [12]. To overcome this stretching of hip flexors and knee extensors was added to the usual physiotherapy regime. The outcome of THR and prosthesis longevity is good, despite the non-physiologic mechanics of the affected hip joint, although these results cannot be compared with any other series in the literature or with non-amputees [5]. Although the risk of fractures is unavoidable in elderly amputees, a high risk of sustaining a fracture in the osteoporotic residual stump should be explained to these patients as a part of health education $[6,10]$.

\section{Conclusion}

Primary hip arthroplasty with distal femur compressive plating and allogenic bone grafting can be used as a definitive management procedure in cases of ipsilateral below-knee amputation with distal femur non-union. Distal femur plating provides absolute stability at the distal femur fracture site and better chances of union with bone grafting. Primary hip replacement allows for satisfactory outcomes, early ambulation, and rehabilitation in such patients.

\section{Clinical Message}

THR with distal femur plating and allogenic bone grafting can be used as a primary and definitive modality of treatment in cases of NOF fractures with distal femur shaft non-union and ipsilateral below-knee amputation.

\section{References}

1. Gustilo RB, Anderson JT. Prevention of infection in the treatment of one thousand and twenty-five open fractures of long bones: Retrospective and prospective analyses. J Bone Joint Surg Am 1976;58:453-8.

2. Moore AT. The self-locking metal hip prosthesis. J Bone Joint Surg Am 1957;39:811-27.

3. Baldwin P, Li DJ, Auston DA, Mir HS, Yoon RS, Koval KJ. Autograft, allograft, and bone graft substitutes: Clinical evidence and indications for use in the setting of orthopaedic trauma surgery. J Orthop Trauma 2019;33:203-13.
4. Braithwaite RS, Col NF, Wong JB. Estimating hip fracture morbidity, mortality and costs. J Am Geriatr Soc 2003;51:364-70.

5. Salai M, Amit Y, ChechikA, Blankstein A, Dudkiewicz I. Total hip arthroplasty in patients with below-knee amputations. JArthroplasty 2000;15:999-1002.

6. Boussakri H, Alassaf I, Hamoudi S, Elibrahimi A. Hip arthroplasty in a patient with transfemoral amputation: A new tip. Case Rep Orthop 2015;2015:593747.

7. Burke MJ, Roman V, Wright V. Bone and joint changes in lower limb amputees. Ann Rheum Dis 1978;37:252-4. 
8. Fraisse N, Martinet N, Kpadonou TJ, Paysant J, Blum A, André JM. Muscles of the below-knee amputees. Ann Readapt Med Phys 2008;51:218-27.

9. Pekmezci M, Nunley RM, Barrack RL. Technique for total hip arthroplasty in a patient with through-knee amputation. J Arthroplasty 2010;25:659.

10. Masmoudi K, Rbai H, Fradj AB, Saâdena J, Boughattas A. Primary total hip replacement for a femoral neck fracture in a below-knee amputee.J Orthop Case Rep 2016;6:63-6.

11. Prickett NM, Scanlon CJ. Total joint replacement in extremities with below-knee amputations. Phys Ther 1976;56:925-7.
12. Mak J, Solomon M, Faux S. Ipsilateral total hip arthroplasty in a dysvascular below-knee amputee for advanced hip osteoarthritis: A case report and review of the literature. Prosthet Orthot Int 2008;32:155-9.

13. Hardinge K. The direct lateral approach to the hip. J Bone Joint Surg Br 1982;64:17-9.

14. Baker RP, Squires B, Gargan MF, Bannister GC. Total hip arthroplasty and hemiarthroplasty in mobile, independent patients with a displaced intracapsular fracture of the femoral neck. A randomized, controlled trial. J Bone Joint Surg Am 2006;88:2583-9.

\section{Conflict of Interest: Nil} Source of Support: Nil

Consent: The authors confirm that informed consent was obtained from the patient for publication of this case report

\section{How to Cite this Article}

Pawar E, Gavhale S, Kambli M, Yadav A, Bansal S, Mishra S. Total Hip Replacement and Distal Femur Shaft Non-union in a Case of Below-knee Amputation - A Case Report. Journal of Orthopaedic Case Reports 2021 February;11(2): 102-106. 